\title{
Ethnologies
}

\section{Les bals de finissants et les initiations universitaires au regard des concepts d'Erving Goffman}

\section{David Harvengt}

Volume 26, numéro 2, 2004

Québec - Ethnologie du proche

Québec - Ethnology At Home

URI : https://id.erudit.org/iderudit/013747ar

DOI : https://doi.org/10.7202/013747ar

Aller au sommaire du numéro

Éditeur(s)

Association Canadienne d'Ethnologie et de Folklore

ISSN

1481-5974 (imprimé)

1708-0401 (numérique)

Découvrir la revue

Citer cet article

Harvengt, D. (2004). Les bals de finissants et les initiations universitaires au regard des concepts d'Erving Goffman. Ethnologies, 26(2), 179-200.

https://doi.org/10.7202/013747ar
Résumé de l'article

Les bals de finissants et les initiations universitaires sont souvent associés à des rites de passage. Il est cependant possible de les analyser sous un autre angle, celui des interactions. Erving Goffman a été un des plus grands théoriciens des rites d'interaction. Son approche lui a permis d'analyser les interactions sociales dans leur aspect routinier. Cet article propose une lecture des bals et des initiations à travers les concepts de Goffman. Qui sont les acteurs en jeu ? Quelles sont les interactions qui se nouent entre les différents groupes dans ces rites ? Quelles relations entretiennent-ils avec leur environnement? 


\title{
LES BALS DE FINISSANTS ET LES INITIATIONS UNIVERSITAIRES AU REGARD DES CONCEPTS D'ERVING GOFFMAN
}

\author{
David Harvengt \\ Université Laval
}

Chaque printemps et chaque automne, le milieu scolaire québécois vit au rythme de deux événements majeurs : les bals de finissants du secondaire et les initiations universitaires. Ces deux événements sont sans aucun doute des rituels, mais peuvent-ils se circonscrire à une seule forme de ritualité ? Ou, au contraire, doivent-ils être appréhendés, selon des angles divers, en tant que rites de passage, rites festifs, ou encore rites d'élévation... voire un amalgame de tout cela ? La ritualité contemporaine influence la compréhension de ces deux rituels par une individualité de plus en plus présente. Les contextes festifs et ludiques y ont aussi un rôle important. Une autre façon intéressante d'appréhender ces deux rituels est l'analyse des rites d'interactions élaborée par Erving Goffman. C'est ce que cet article va tenter de démontrer à travers une lecture "goffmanienne » des bals de finissants et des initiations universitaires.

La relation entre les rites d'interactions et les deux rituels au cœur de mon analyse tient en deux points principaux qui se recoupent : la mise en scène et la métaphore théâtrale. Mise en scène subtile de la vie quotidienne chez Goffman, et mise en scène sans doute plus éclatante dans les bals et les initiations, où les acteurs sociaux jouent à l'évidence un rôle hors de leur quotidien. Ce rôle, d'ailleurs, nous amène à la métaphore théâtrale utilisée par Goffman, qui est pertinente pour la présente analyse. Une lecture de ces deux événements sous la loupe goffmanienne suppose néanmoins quelques ajustements par rapport à l'analyse des rites d'interactions que propose le sociologue américain. 
En effet, Goffman propose une approche intimiste de la relation, ou plutôt de l'interaction, entre les acteurs ou les groupes d'acteurs (ce qu'il appelle les équipes). C'est ce cas qui retiendra davantage mon attention. Bien qu'il soit tout à fait possible d'analyser les initiations et les bals en tant qu'interactions individuelles (ce qui serait même très intéressant à réaliser), je les aborde, pour ma part, en tant que rites collectifs et donc en tant qu'interactions collectives. De plus, avec cette lecture, je quitte une analyse appliquée surtout à des activités ordinaires ou routinières, telle que l'avait fait Goffman lui-même, pour examiner des événements singuliers, à tel point qu'ils ne se présenteront souvent qu'une fois ou deux dans la vie des acteurs. Cela m'oblige à une lecture quelque peu différente de celle proposée par le sociologue américain, tout en considérant que ses concepts théoriques demeurent pertinents.

Pour présenter ses concepts, Erving Goffman utilise la métaphore théâtrale, où les individus deviennent des acteurs qui évoluent dans des régions postérieures (coulisses) et des régions antérieures (scène) où ils produisent une représentation dans un décor donné. Ces représentations donnent une définition d'une situation d'interaction. Les acteurs ont le devoir de préparer leur représentation et de l'ajuster aux circonstances de la représentation qui parfois peuvent leur échapper en partie. Cette approche présente les individus sociaux comme des acteurs dirigeant leurs relations avec autrui, même si le processus est souvent tellement imprégné dans l'attitude des acteurs qu'ils n'y prêtent pas une réelle attention.

Avant d'analyser ces deux rituels, il est bien sûr indispensable de donner, ne serait-ce que brièvement, chacun des deux schémas qui structurent, pour l'un, la plus grande partie des bals de finissants, pour l'autre, les initiations universitaires.

Cet article évoque donc tout d'abord brièvement le déroulement des bals de finissants et des initiations universitaires, tels que je les ai observés durant le terrain d'enquête et selon les témoignages des informateurs que j'ai rencontrés. J'analyse ensuite ces événements en prenant appui sur les théories de Goffman. Mais ce texte n'est bien sûr qu'un survol d'une lecture goffmanienne des bals de finissants et des initiations universitaires, qui mériteraient à eux seuls un travail de plus large envergure. 


\section{Le déroulement des bals de finissants du secondaire}

Au Québec, le bal de finissants se déroule à la fin de la cinquième et dernière année du secondaire. Il marque la fin de ce cycle avant que les élèves ne poursuivent un parcours sur une autre voie, scolaire ou non. Le bal a lieu en général vers la fin du mois de juin, lorsque l'année scolaire et les examens sont terminés. Il se découpe en trois moments différents : l'avant-bal, le bal lui-même et l'après-bal. Mais cette journée mémorable ne peut avoir lieu sans la préparation qui la précède, une préparation à la fois individuelle et collective. Les finissants ont en effet bien des choses à prévoir dans les mois précédant le bal : quels habits vont-ils revêtir? Quel sera leur moyen de transport pour se rendre au bal (limousine, voiture de sport, voiture familiale, etc.) ? Seront-ils accompagnés ou non? L'habillement occupe ici une place privilégiée et le choix ne se fait pas toujours au premier essayage.

On est allés une dizaine de fois essayer, je pense, quinze robes de la shot. Nous autres, c'était notre trip. On voulait être belles, on voulait que la soirée soit parfaite. Pis dès le début du secondaire 5 , on en parlait, on avait hâte au bal (E.L., finissante ${ }^{1}$ ).

Les finissants sont rarement laissés à eux-mêmes dans cette démarche, tout individuelle qu'elle soit. Les parents jouent parfois un rôle important dans ce choix, autant pour les garçons que pour les filles: "J'ai été dans un magasin avec mon père acheter un complet, pis c'est moi qui l'ai choisi ", affirme un finissant (P.M.). Pour la plupart des filles, si le choix et l'achat de la robe se font en compagnie de la mère, l'avis des meilleures amies est également parfois requis (E.L.). Les parents assument aussi une part financière importante en payant souvent une partie ou la totalité de la robe ou de l'habit. L'importance accordée au moyen de transport ou à l'accompagnateur ou l'accompagnatrice dépend davantage de chacun des finissants.

Ça c'est super important dans les mentalités de certaines personnes.

J'ai une amie, elle, pas être accompagnée, ça aurait été un drame. Alors elle a commencé à y penser au début de l'année, pis en février c'était fait. Mais c'était la moitié qui était accompagnée (J.M.L.).

Cette préparation individuelle n'exclut pas, loin de là, une préparation collective qui met en place toute l'organisation du bal lui-

1. Pour des raisons de confidentialité, nous utilisons uniquement les initiales des informateurs et informatrices. 
même. Ainsi, certains finissants s'impliquent dans différents comités afin d'organiser les soirées (bal, après-bal) et de préparer tout ce qui entoure cette fin de cycle (albums de finissants, par exemple). La préparation du bal lui-même peut être ingrate ; c'est un travail de longue haleine qui passe presque inaperçu. "Ça paraît pas, parce que quand les élèves arrivent, ils ne se demandent pas tous qu'est-ce qu'on a fait. Mais c'est énormément de préparation. Il y a beaucoup de choses à penser" explique une organisatrice (V.D.). Ces comités veillent aussi au financement du bal. Pour faire mousser les ventes des élèves, certains organisateurs trouvent des stratagèmes : ils réalisent des thermomètres en carton pour chaque classe sur lesquels les ventes de chacune sont indiquées. À la fin de la campagne, celle qui aura rapporté le plus d'argent gagne un dîner pizza. Le comité de l'après-bal est souvent plus marginal que celui du bal; il n'est pas toujours reconnu par la direction scolaire et il compte moins de membres (deux ou trois plutôt qu'une dizaine).

Finalement, après ces longs mois de préparation, voici enfin le grand jour ! La matinée et le début de l'après-midi sont réservés à la préparation individuelle : coiffure, maquillage, habillement. Ces préparatifs sont souvent plus longs pour les finissantes que pour leurs homologues masculins. L'avant-bal a lieu en fin d'après-midi, le jour même du bal. Il rassemble de petits groupes de finissants (de quatre à cinq jusqu'à une vingtaine) et quelques-uns de leurs proches (parents, amis). Il peut y avoir des variantes d'un avant-bal à l'autre. En réalité, si de plus en plus de finissants vivent un avant-bal, il n'en reste pas moins que c'est aussi le moment de l'événement qui est le moins courant ; certains finissants n'en ont tout simplement pas. L'avant-bal se déroule chez les parents d'un des élèves et il est essentiellement organisé par les finissants euxmêmes. Il consiste la plupart du temps à prendre un verre et des photos en comités relativement restreints. "Prendre un apéritif, prendre des photos avec tes amis, avec tes parents. Ben c'est un moment aussi avec tes parents, parce qu'au bal, c'est plus avec les amis » (C.B.).

Le bal lui-même commence aux alentours de $18 \mathrm{~h}$ ou $19 \mathrm{~h}$. Le déplacement jusqu'au lieu du bal est un des moments publics les plus évidents de l'événement; certains finissants en profitent donc pour s'afficher, se montrer. Malgré tout, le moyen de transport varie d'un finissant à l'autre. Une chose est sûre néanmoins, plus la voiture sera luxueuse, plus les finissants voudront s'afficher. 
On est descendus en limousine [...]. On est allés sur la rue Cartier, sur la Grande Allée pour aller se vanter un peu, disons. On était huit dans la limousine... on est allés parader (S.C.).

Mais nul besoin de limousine pour annoncer ses couleurs.

On avait fait décorer l'autobus plein de banderoles « gradués ». Fait que là on sortait toujours les mains dehors, on criait à tout le monde, on chantait, on avait bien hâte d'arriver. Il y avait pas mal d'énervement (V.D.).

La transition entre l'arrivée sur le lieu du bal, en général un hôtel, et son début est marquée par la consommation d'un cocktail offert à tous les convives. C'est un temps où les finissants, leurs accompagnateurs, leurs parents et leurs professeurs sont réunis. C'est aussi le premier moment où les finissants sont tous ensemble.

On est restés avec nos amis [...]. Il y a des photos de groupe, on se promène $[. .$.$] . Première affaire, on parle de l'habillement, parce que$ si t'en parles pas c'est que tu trouves ça laid et que t'oses pas le dire (J.M.L.).

Les personnes présentes durant les différentes étapes du bal varient. Cependant, dans tous les cas, les accompagnateurs, les professeurs et la direction scolaire sont présents pendant tout le bal. Par contre, en ce qui concerne les parents, plusieurs cas de figure sont possibles: ils peuvent être présents durant le bal, durant une partie de la soirée (le plus souvent au souper ; ils s'en vont avant la soirée dansante) ou encore ne pas être présents du tout. Le nombre de finissants est un des facteurs qui influencent la présence ou non des parents ; plus il y a de finissants, moins les parents seront présents, compte tenu de la capacité des salles de bal. La soirée comprend plusieurs activités, dont l'agencement peut varier : le souper, la remise de prix $^{2}$, la diffusion d'une vidéo ${ }^{3}$ et la soirée dansante en sont les principales. Cette dernière est souvent relativement brève : elle dure une à deux heures maximum.

Le souper se termine vers dix heures. Pis là, vers onze heures, même onze heures quinze, le monde commence à se changer déjà. Dès que ça commence à danser, il y en a plein qui restent pas dans la salle (J.M.L.).

2. Ces prix sont par exemple : le plus beau sourire, le plus beau couple... Ils peuvent être teintés d'humour : le ou la gagnant(e) du plus beau sourire reçoit un bâton de baume pour les lèvres.

3. C'est souvent une vidéo filmée durant l'année scolaire et qui présente un peu le quotidien des finissants. Il s'agit souvent d'un moment fort de la soirée. 
Aux alentours de minuit ou d'une heure du matin, les finissants se mettent en route pour l'après-bal, soit directement du lieu du bal, soit après un détour par l'école. Ils ont tous ôté leurs beaux habits de bal pour en revêtir d'autres beaucoup plus confortables et chauds. Le trajet en autobus jusqu'à l'endroit où se déroule l'après-bal dure généralement entre une demi-heure et une heure. L'après-bal a lieu le plus souvent dans des endroits en dehors de la ville; les érablières semblent avoir la cote auprès des organisateurs. L'après-bal est le moment le plus festif de la journée. L'ambiance est d'ailleurs déjà joyeuse durant le trajet qui conduit les finissants vers le lieu de la fête.

Dans l'autobus, il y avait des chansons. Il y en avait qui avait déjà bu ou fumé. C'était pas mal énervé mais c'était le fun. Il y en a qui dormaient mais tout le monde était pas mal dedans. Il y en a qui ont commencé Star Académie, ils se sont fait crier dessus, alors ils ont arrêté (J.M.L.).

Lors de l'après-bal, les finissants sont seuls (souvent avec les accompagnateurs, mais pas toujours) ; les parents et le personnel scolaire ne sont plus présents. Malgré tout, de plus en plus d'après-bals sont organisés par des entreprises spécialisées avec pour conséquence la présence d'agents de sécurité et d'ambulanciers. Ils établissent parfois un périmètre de sécurité autour des bâtiments pour éviter que les finissants ne s'éloignent dans les bois avoisinants. Cela n'empêche pas pour autant les finissants de fêter, boire, danser, discuter et parfois consommer quelques produits illicites.

Moi j'ai trouvé ça plus le fun que le bal. C'est plus ça un party de fin d'année. Ça, ça montrait vraiment qu'on fêtait la fin de notre secondaire. Il y a du monde qui criait : « c'est fini, êtes-vous contents ? » et tout le monde gueulait (J.M.L.)

Plusieurs autobus sont prévus pour le retour en ville à diverses heures de la nuit. Ceux qui ont le moins supporté les aléas de la fête sont souvent les premiers à repartir. Le dernier autobus part en général aux petites heures du matin (six ou sept heures). L'autobus dépose généralement les finissants à l'école où les parents viennent chercher les fêtards. Les finissants se quittent souvent sans que la fin du bal ne soit spécialement marquée, sinon par quelques embrassades. Toutefois, l'émotion surgit parfois, surtout lors des derniers départs. "Dans l'autobus, le monde ça pleurait, c'était vraiment triste. Il y avait des amis qui jouaient de leur guitare, les vieilles chansons qu'on jouait » 
(E.C.). D'autres amies fondent en larmes en se disant qu'elles ne se reverront plus jamais... au bout du compte, elles habitent toujours toutes deux à Québec et se voient encore, raconte une finissante (S.C. $\left.{ }^{4}\right)$.

\section{Le déroulement des initiations}

À l'inverse des bals de finissants, les initiations universitaires s'étalent sur plusieurs jours. Les initiations scolaires à l'Université Laval, qui a constitué mon terrain d'observation, se déroulent la première semaine de la rentrée, soit au tout début du mois de septembre ${ }^{5}$. Quelques rares activités indirectement reliées aux initiations peuvent avoir lieu la ou les semaines suivantes comme un "vin et fromage », par exemple. Tout comme les bals de finissants, les participants aux initiations doivent se préparer: les initiés se préparent individuellement selon les consignes qu'ils reçoivent des initiateurs et ces derniers organisent toute l'initiation. L'organisation d'une initiation peut demander plusieurs mois de préparatifs. En médecine, par exemple, plus d'une centaine de nouveaux se font initier chaque année et presque autant d'anciens participent à l'initiation à divers degrés. Ainsi, dès le mois de mars, deux présidents sont élus et ce sont eux qui vont chapeauter la trentaine de responsables de comités qui organisent les différentes activités. Les organisateurs doivent également se conformer aux exigences de l'université et remettre un programme détaillé de l'initiation qu'ils proposent au doyen de la faculté. L'initié, lui, reçoit en général dans le courant du mois de juillet un document informatif sur l'initiation. Celui-ci peut prendre diverses formes : cahier volumineux, feuillets, ou sites Internet. L'initié y trouve ce dont il a besoin pour préparer son initiation. Ainsi, le nouveau passe beaucoup de temps à confectionner son costume, à trouver les objets requis, à apprendre les chansons. Il y a derrière cette préparation une mise à l'épreuve de l'étudiant : est-il débrouillard ou, à tout le moins, rusé ? Arrivera-t-il à trouver tous les objets? À faire un costume décent? Le costume est un élément commun à la plupart des initiations universitaires. Il est associé au thème de l'initiation du programme (le thème en médecine en 2002 était Mario Bros; en actuariat, en 2002,

4. J'ai présenté ici un schéma très général du bal de finissants ; il va de soi que des variantes existent mais il s'agit néanmoins d'un canevas qui semble se reproduire souvent.

5. Il n'y a pas vraiment d'initiation pour la rentrée hivernale. 
c'était les «Pierrafeu ») ou au programme d'études (en pharmacie, pour l'initiation 2000, le costume était une bouteille de Motrin ; en médecine dentaire, en 2000, il s'agissait de la "fée des dents $\left.{ }^{6}\right)$. Ce costume, les initiés le revêtent le matin de l'initiation pour se rendre sur le campus. En ces heures matinales, les initiés se regroupent près de « leur » pavillon, formant des grappes costumées; le campus prend des allures de carnaval ou de champ de bataille.

J'ai vraiment aimé ça quand t'arrives sur le campus. Ça m'a vraiment fait penser à la guerre, à la limite. Ça flashait vraiment comme ambiance, parce que t'avais plein de masses de gens tous costumés différent. T'sais comme tout une masse de rose, tout une masse de jaune, une masse de vert, comme des îlots. Comme justement la guerre, comme une espèce de grand champ avec différents groupes ou tribus qui sont là. L'appartenance au groupe avec le costume était évidente (A.M.D.).

À cet instant, l'initiation débute. À l'instar des bals de finissants, plusieurs activités semblent constantes d'une initiation à l'autre, même si leur agencement dans les journées d'initiation varie. On pourrait classer ces activités selon qu'elles se déroulent sur le campus ou en dehors. Le plus souvent, les animations sur le campus sont celles qui se déroulent les premiers jours de l'initiation. L'accueil des nouveaux est la première d'entre elles. Il est souvent ponctué d'un workout humoristique très populaire auprès des différents organisateurs. Cet accueil constitue le premier contact physique entre nouveaux et anciens. La deuxième activité la plus répandue est la «visite » du campus où les initiés disputent quelques épreuves bon enfant devant les principaux pavillons. Pour ces épreuves, les nouveaux sont donc répartis en équipes sous la tutelle d'anciens. L'appartenance à une équipe est le fruit du hasard. En recevant son cahier d'initiation en juillet, l'initié apprend par la même occasion quelle sera son équipe. Les organisateurs ne se donnent que quelques rares consignes, comme une parité de nouveaux. Dans certains programmes qui comprennent une majorité de filles, il peut s'agir d'avoir un nombre égal de "gars » par équipe. Car «pour les jeux de boisson, souvent les gars embarquent plus, ils en prennent plus » annonce un organisateur (S.B.).

Les activités en dehors du campus se scindent en deux catégories: d'une part les soirées en ville qui peuvent avoir lieu dans des bars ou

6. Collection Martine Roberge et Karine Laviolette, ms 1. Archives de folklore et d'ethnologie de l'Université Laval. 
dans des salles privées (il faut signaler que certaines soirées ont lieu sur le campus même) et, d'autre part, la journée en dehors du campus qui est le point culminant des initiations. Pratiquement toutes les initiations comprennent une ou plusieurs soirées dans un bar. Ces soirées ressemblent souvent à une soirée " ordinaire », ponctuée de quelques jeux dont les populaires «calages» de bière. En médecine, une soirée intitulée le «festival du plombier » conclut la première journée d'initiation et comprend plusieurs épreuves, dont le très attendu century ${ }^{7}$, qui occupe une grande part des discours des anciens pendant toute la journée. La journée hors-campus se déroule le plus souvent lors de la dernière journée d'initiation. Elle se tient toujours sur des sites situés en dehors de la ville, comme des campings, des camps, etc. En général, le hors-campus commence en début d'après-midi. À l'instar des après-bals, le déplacement se fait en autobus et le lieu est tenu secret, au moins pour les initiés. Plusieurs autobus sont également prévus pour le retour des initiés et initiateurs, les premiers en fin de soirée (vers vingt-deux ou vingt-trois heures) et les derniers dans le courant de la nuit (deux ou trois heures du matin). Le hors-campus se caractérise essentiellement par des épreuves de type physique, de consommation d'alcool et de maculage. À la fin des épreuves, les initiés affichent donc tous une belle couleur brune, de la tête aux pieds ${ }^{8}$. Aussi doivent-ils se laver avant de poursuivre la soirée. Dans l'initiation de médecine, cet épisode du décrassage est particulièrement évocateur. Les nouveaux vont se laver dans un lac. Aux abords de celui-ci, chacun enlève ses vêtements sales et les dispose dans un tas avant d'aller se baigner en maillot de bain. Enlever ses habits sales est un geste symbolique fort. Par cet acte, on change de «peau », on délaisse sa deuxième " peau »", celle de novice. Un initié souligne d'ailleurs l'importance de ce moment.

À la fin du hors-campus, on allait se baigner dans le lac pour se laver. Je pense que quand on retournait dans la salle en habit, en vêtements normaux pour souper tout le monde ensemble, pour moi, c'est ça qui a marqué : «maintenant vous êtes initiés ». À partir du moment où on enlève notre costume puis qu'on s'habille en civil, là on redevient égaux à tous ceux qui étaient en civil jusqu'à maintenant (F.D.).

7. Au cours duquel trois participants par équipe (dont au moins une fille) doivent boire une once (environ $30 \mathrm{ml}$.) de bière par minute durant 60 minutes.

8. Il va de soi que certaines initiations sont moins salissantes que d'autres.

9. «Ce T-shirt s'agencera à merveille avec votre boxer à la journée sportive et sera votre deuxième peau pour toute la semaine ", écrivent les organisateurs dans le cahier d'initiation. 
Au milieu de la soirée, vers vingt-deux heures, les premiers autobus repartent vers Québec. Ils sont pleins. Les participants sont fatigués, et seuls les plus motivés et les organisateurs restent pour la soirée qui se terminera vers trois heures du matin. Le voyage de retour signale souvent la véritable fin de l'initiation, sinon dans les faits, au moins dans les esprits. À partir de cet instant, la vie ordinaire de l'étudiant (re)prend son cours.

À côté de ces initiations «classiques », certains organisateurs tentent de trouver d'autres formules. Tel fut le cas pour le programme de sociologie en 2002. C'est ce que nous explique son principal organisateur.

On a loué un chalet, c'était quelque part dans le bois. Il nous fallait un local, pis on voulait pas faire ça sur le campus. [...] On a acheté de la bière et de la nourriture. [...] L'idée c'était qu'il y ait pas de jeux ou n'importe quelle niaiserie qu'on retrouve dans les initiations, genre : une image de la position sexuelle pis des trucs dans le genre. On voulait rien savoir, on voulait rien de dégradant. Pis c'est ce qu'on a réalisé : il y avait de la bière en quantité raisonnable, puis il y avait pas d'entonnoir pis de tuyau, le monde allait s'en chercher quand il voulait. On pouvait jaser avec les nouveaux, faire connaissance. Lidée c'était qu'ils s'intègrent, mais pas pour ce qu'on leur fait faire, mais pour ce qu'ils sont eux-mêmes (M.L.).

Ce discours s'oppose résolument aux initiations. Les arguments ne sont pourtant pas neufs et ils sont même plutôt récurrents ; ils reviennent régulièrement, chaque année, dans les propos des opposants aux initiations. Les principaux arguments «anti-initiation » sont l'humiliation, la dégradation, la vulgarité (par des mimes d'actes sexuels, entre autres) et la beuverie ${ }^{10}$.

\section{Les équipes}

Le déroulement des deux événements, brièvement décrit ci-dessus, nous amène à prendre conscience des différents groupes d'acteurs qui s'y côtoient. Pour mieux comprendre l'implication de ces groupes, il est intéressant d'utiliser plusieurs concepts empruntés à Erving Goffman. Le concept d'équipe est le premier à retenir mon attention. Cette notion,

10. Tout comme pour les bals, il s'agit ici d'un très bref aperçu de ce qui se déroule lors des initiations universitaires. 
qui rejoint un peu celle de groupe, voire celle de rôle, est au centre de la lecture que je propose, dans le cadre de cet article, puisque c'est bien la relation entre les différentes équipes qui détermine en grande partie ma compréhension des deux rituels que sont les bals et les initiations.

Goffman définit le concept d'équipe de la façon suivante :

[u]ne équipe peut donc se définir comme un ensemble de personnes dont la coopération très étroite est indispensable au maintien d'une définition donnée de la situation. C'est un groupe qui est en relation, non pas avec une structure sociale ou une organisation sociale, mais plutôt avec une interaction ou une série d'interactions dans laquelle on maintient la définition adéquate de la situation (1973a : 102 $103)$.

Dans les bals de finissants et dans les initiations universitaires, il y a donc plusieurs équipes différentes qui interagissent entre elles. Dans les bals, les équipes impliquées sont : les finissants, les parents, le personnel de l'école (surtout les professeurs et la direction), les spectateurs et les spécialistes. Ces derniers, selon Goffman, sont « des gens qui se spécialisent dans la construction, la remise en état et l'entretien du spectacle que leurs clients donnent aux autres » (1973a: 148). Dans les bals, ils correspondent au personnel de l'hôtel, aux chauffeurs, aux coiffeurs, etc. Les équipes des initiations universitaires sont : les initiés ${ }^{11}$, les initiateurs, l'administration universitaire ${ }^{12}$, les autres groupes d'initiés et les spectateurs. Les directions scolaires ${ }^{13}$ jouent un rôle similaire dans les deux événements ; il est en de même pour les spectateurs. Ces derniers représentent un groupe extérieur à l'événement, mais qui l'observe. Toutefois, dans les initiations, les spectateurs assument parfois un rôle plus actif dans le rituel en participant (souvent involontairement) à certaines épreuves, comme par exemple, lorsqu'un(e) initié(e) doit draguer un(e) inconnu(e). Les directions scolaires occupent une place prépondérante dans la préparation des rituels en exerçant une forme de contrôle, en établissant certaines balises dans le déroulement des événements.

11. Le terme "initié » est employé comme synonyme de «novice " même si, a priori, les initiés sont ceux qui ont déjà vécu leur initiation. Si je l'utilise de cette façon, c'est qu'il s'agit du terme souvent employé par les acteurs euxmêmes pour désigner les nouveaux.

12. J'ajoute ici la CADEUL (Confédération des associations des étudiants de premier cycle de l'Université Laval) qui s'implique dans l'encadrement des initiations.

13. Les directions du secondaire, mais également l'administration universitaire. 
À partir de ce concept d'équipe, il est possible d'établir une distinction entre les bals et les initiations. En effet, dans les premiers, les finissants sont l'équipe principale, tandis que dans les initiations il faut tenir compte à la fois des initiés et des initiateurs. Ces trois groupes sont considérés comme les équipes principales qui se définissent par rapport à la représentation, c'est-à-dire à l'activité d'un ou de plusieurs acteurs devant un public qui fonde une situation donnée. Dans les initiations universitaires, c'est bien la confrontation entre initiés et initiateurs, aussi amicale qu'elle soit, qui est au cœur de l'événement, qui le définit.

\section{Définition des équipes}

Il est malheureusement impossible ici de définir l'ensemble des équipes, aussi me concentrerai-je sur les trois principales évoquées cidessus. Chaque équipe a en effet ses propres caractéristiques et occupe une place spécifique dans le rite. Ainsi, l'équipe «finissant» ne prend réellement corps qu'au moment où le bal débute, avec des prémices parfois à l'avant-bal. Cette équipe est le fruit d'une part de l'équipe " élèves » ${ }^{14}$ qui lui préexiste et, d'autre part, du contexte particulier du bal. En fait, ce dernier, par ses côtés cérémoniel et festif, transforme l'équipe «élèves » (par l'habillement entre autres). Les individus de l'équipe «finissants » maintiennent malgré tout souvent une image qui correspond au rôle qu'ils tenaient dans l'équipe « élèves ». Par exemple, un élève qui était extraverti durant l'année scolaire le sera probablement aussi lors du bal, avec peut-être un côté extravagant en plus ; à l'inverse, un élève timide le demeurera sûrement, avec peut-être un laisser-aller plus prononcé. Il y a quand même parfois des surprises. «Il y en a que oui c'était surprenant. Tu t'attendais pas que cette personne-là fasse ça, c'était plus low profile durant l'année » confie un finissant (P.M.). Les finissants ont aussi la caractéristique de ne vivre «leur» bal qu'une seule fois, hormis quelques rares exceptions. Ce qui ne veut pas dire que certains d'entre eux n'auront pas l'occasion d'en vivre plus d'un (parfois la même année), mais ce sera alors en tant qu'accompagnateur.

Les initiateurs, à l'instar des finissants, se connaissent avant l'initiation. Ce sont également eux qui organisent l'initiation. C'est d'ailleurs un des points clés des initiations universitaires : les anciens contrôlent la représentation et, jusqu'à un certain point, le décor. En effet,

14. Cette équipe qui se bâtit au fil des cinq années du secondaire. 
contrairement aux nouveaux, ils connaissent les lieux et ils décident où se dérouleront les différentes activités, dans les limites imposées par l'administration universitaire. Toutefois, à la différence des finissants, les initiateurs ont déjà vécu «leur» initiation. Leur position dans le rite s'est transformée. Les initiés, quant à eux, forment une équipe particulière puisque ses membres ne se connaissent pas, à part quelques exceptions, avant l'événement. Le groupe ne prend véritablement corps qu'aux premiers instants de l'initiation, lorsque les nouveaux se regroupent sur le campus. Ce groupe n'est donc pas le résultat d'interactions qui préexistent à l'initiation. C'est même le contraire, puisque c'est le but avoué de cette dernière de provoquer ces interactions en plaçant les nouveaux dans une situation inaccoutumée où le groupe peut davantage se cimenter, à tout le moins plus rapidement, que dans un contexte quotidien. Toute la préparation des initiés est donc individuelle, sans être pourtant personnelle, puisqu'ils doivent suivre les instructions des initiateurs. Les initiés ont donc peu, sinon aucun, contrôle de la représentation. Ils trouvent cependant certaines façons de palier cette lacune : la non-participation est en une, la surenchère sur l'épreuve demandée en est une autre. Lorsqu'un initié effectue un strip-tease (jusqu'au caleçon) en plein midi dans une cafétéria de l'université, alors qu'il lui était simplement demandé de draguer une inconnue, il a sans doute l'impression d'exercer un certain contrôle sur sa représentation ${ }^{15}$.

\section{Les rites de ratification}

Quels sont les rapports qu'entretiennent ces équipes principales avec les autres équipes qui les entourent dans l'événement? L'attitude des autres groupes, en particulier vis-à-vis des équipes principales, permet une meilleure définition de la situation et les équipes principales ne sont donc pas les seules à la définir. Pour essayer de comprendre ces relations, j'utilise un autre concept d'Erving Goffman, celui des rites de ratification.

Il existe des «rituels de ratification » accomplis pour et envers un individu dont le statut s'est altéré d'une certaine façon, dont les relations, l'aspect, le rang, la qualification, bref, les buts et les espoirs ont changé. Les rituels ratificatoires expriment que leur exécutant est

15. Encore qu'il faille tenir compte ici de l'influence du groupe sur le comportement des individus et penser que ce comportement vient aussi de la volonté de se faire remarquer. 
sensible à la situation de celui qui a subi un tel changement, qu'il désire poursuivre la relation avec lui et lui conserver son appui, et qu'en fait les choses sont ce qu'elles étaient en dépit de la transformation reconnue (1973b: 77-78).

Ces rites sont donc à la fois une reconnaissance du changement et l'affirmation que le changement ne modifie pas la relation. Ainsi, les bals de finissants sont la reconnaissance du changement vécu tant par soi (les finissants) que par les proches (parents, professeurs, etc.). De ce fait, le bal, tout en restant l'apanage des finissants, se trouve en somme au centre des interactions entre les élèves, les parents et le personnel de l'école. En effet, sans les groupes qui gravitent autour des finissants, le bal n'aurait pas lieu, en tout cas pas sous sa forme actuelle. Il est donc permis - et d'une certaine façon partiellement contrôlé - par ces autres groupes, à travers l'organisation, la dépense, etc. Ainsi par exemple, les parents ont une attitude beaucoup plus active lors de la préparation individuelle du finissant et beaucoup plus passive durant l'événement lui-même. Ce comportement amplifie davantage encore la position privilégiée des finissants en cette journée de bal. Ce qui vient un peu en contrepoids de ce qu'affirmait Goffman, pour qui, lorsque deux équipes sont en interaction, l'une se trouve en position de supériorité et l'autre tente de diminuer la distance entre les deux (1973a : 189). Ici, au contraire, les équipes qui entourent celle des finissants accentuent d'une certaine façon cette distance. Il y a donc une valorisation des finissants, même si les bals ne sont pas des rites d'inversion : il n'y a pas ici de changement de position entre finissants et adultes. Par ailleurs, le deuxième aspect du rite de ratification, soit celui qui affirme que le changement ne modifie pas la relation, n'a pas lieu d'être dans le bal, puisque le groupe se dissout à la fin du bal.

Dans le cas des initiés, la reconnaissance n'est pas faite par les proches mais par le nouveau milieu (universitaire en l'occurrence) dans lequel les initiés revendiquent une nouvelle place. La confirmation que ce changement ne modifie pas la relation n'existe pas non plus, puisque le groupe ne se connaît pas avant l'événement. Pour les initiateurs, leur changement de statut est encore plus symbolique et ce sont presque davantage eux-mêmes, plutôt que d'autres, qui se congratulent à travers l'initiation. Après tout, même si l'initiation se définit pour beaucoup à partir de la relation entre initiés et initiateurs, ces derniers ne sont pas le pôle d'attraction de l'événement. 
En définitive, si un seul changement est au cœur des bals de graduation, celui des finissants, il y a dans les initiations universitaires un double changement, celui des initiés qui deviennent de nouveaux étudiants et celui des initiateurs qui, grâce à l'arrivée de " nouveaux » nouveaux, deviennent enfin de «vrais » anciens. Mais le côté volontaire de ces rites atténue ces changements dans le sens où, même sans bals de finissants ou sans initiations, chaque individu peut vivre la fin du secondaire et le début de l'université avec autant d'intensité que les participants à ces événements.

\section{Les régions}

Toutes ces équipes évoluent dans un décor spécifique que Goffman précise à travers la notion de région. Il distingue deux régions, la région antérieure et la région postérieure. La région antérieure est simplement l'endroit où se déroule la représentation en tant que telle. Le sociologue américain s'étend davantage sur la région postérieure, qu'il nomme aussi coulisses. Cette région est «[...] un lieu, en rapport avec une représentation donnée, où l'on a toute latitude de contredire sciemment l'impression produite par la représentation» (1973a: 110). C'est l'espace tout indiqué pour mettre au point la représentation, rassembler les éléments scéniques, réajuster, voire éliminer les rôles des différents acteurs, etc. Enfin, selon Goffman, «c'est là que l'acteur peut se détendre, qu'il peut abandonner sa façade, cesser de réciter un rôle, et dépouiller son personnage » (1973a: 111). Pour prendre un exemple, la région antérieure serait la salle à dîner d'un restaurant et la région postérieure, la cuisine. La région postérieure est souvent interdite au public et s'il y accède sans permission, cela peut provoquer un malaise important. Parfois les acteurs utilisent une partie de la région antérieure en guise de région postérieure ; ils adopteront pour ce faire le comportement propre aux coulisses. Pour prendre un exemple relié à la restauration une fois encore, lorsque des employés d'un restaurant s'installent à une table de la salle à dîner et y adoptent un comportement utilisé d'habitude en coulisses, ils étendent alors ces dernières à la région antérieure où ils se trouvent. Or, dans le cadre particulier des bals de finissants et des initiations universitaires, ces régions subissent une forme de brouillage dû au contexte festif de ces événements. Ainsi, là où le théâtre sépare scène et coulisses, voire même la salle, la fête, elle, «crée une interaction confuse entre ces différents éléments» (Piette 1988: 195). Autrement dit, scène et coulisses se mêlent. Ce contexte festif amène un 
fléchissement de certaines limites, quoique l'attitude de chacun soit toujours assujettie à son propre rôle (finissant, parent, initié, etc.). Les finissants peuvent ainsi adopter une attitude d'autant plus décontractée qu'ils sont les « rois » de la fête. Nous assistons donc à un agencement des limites ordinairement admises : les finissants, souvent mineurs, ne peuvent pas légalement acheter de l'alcool, mais ils n'éprouvent aucune difficulté à en acquérir ce jour-là. La flexibilité des limites amène donc un débordement des coulisses dans la scène, ou de la région postérieure dans la région antérieure. Dans le bal et dans l'initiation, la structure même de ces régions varie selon les différents moments des événements. Ainsi, la préparation de l'élève pour le bal se fait en région postérieure, soit dans la maison familiale du finissant, région qu'il partage non pas avec ses équipiers finissants mais avec ses proches ${ }^{16}$. Une partie de cette région postérieure peut cependant être agencée en région antérieure pour la tenue de l'avant-bal. Durant le trajet qui amène les finissants jusqu'à l'hôtel, la voiture est un élément du décor dans la région antérieure qu'est la voie publique. Ne pourrait-elle pas être la scène à partir de laquelle les finissants offrent leur représentation, en exultant par les fenêtres ou le toit ouvrant, en criant, ou tout simplement en s'offrant à la vue des spectateurs? Quoiqu'il en soit, la voiture participe pleinement à la définition de la situation : ceci est un bal de finissants. Enfin, l'hôtel a ses propres régions antérieures (le hall, la salle de bal, etc.) et ses régions postérieures, la plupart interdites, hormis au personnel de l'hôtel. Les toilettes constituent un cas à part puisqu'elles sont la seule partie de la région postérieure à être accessible aux autres groupes. Elles ont aussi une double fonction - hormis celle naturelle : elles permettent à la fois de réajuster son apparence et elles sont parfois le lieu de la préparation de l'après-bal.

T'as un gros ramassis dans la salle de bain... c'est débile, tout le monde se change, ben il y a l'entrée de la salle de bain et les toilettes, alors on peut même pas se changer aux toilettes. Tout le monde se change devant les autres en plein milieu de la salle de bain (J.M.L.).

L'après-bal est en soi un cas particulier : correspond-il à une région antérieure ou postérieure ? Une chose est sûre, il y a un changement de représentation puisque l'après-bal se fait d'abord et avant tout pour soi, pour les finissants sans autres spectateurs, les parents et les professeurs

16. Et à ce moment, on peut se demander si le finissant fait déjà partie de l'équipe «finissants» ou s'il ne fait pas plutôt partie de l'équipe «famille». 
n'étant plus présents. L'après-bal est aussi le moment où comportements de coulisses et comportements festifs sont les plus importants. Pourtant, il y a bien une représentation, c'est-à-dire que le site de l'après-bal peut très bien se comprendre comme une région antérieure. L'après-bal est un bel exemple de cette confusion, du brouillage entre région antérieure et région postérieure dans un contexte festif. L'accessibilité aux régions n'est qu'un facteur qui permet de comprendre les différentes équipes. Dans les bals, un autre facteur entre en ligne de compte ; c'est l'attitude des acteurs dans ces régions selon leur équipe d'appartenance. En effet, les finissants sont beaucoup plus mobiles que les parents, les professeurs et les spectateurs. Tout au long du bal, certains d'entre eux vont et viennent : ils évoluent à leur gré dans les régions antérieures de l'hôtel, ce que ne font pas, ou font moins, les membres des autres équipes.

La situation est identique dans les initiations universitaires. Les différents groupes interagissent dans ces régions, certaines étant accessibles à l'un ou à l'autre, tandis que d'autres régions sont les lieux où tous se croisent. En fait, dans les initiations, les régions antérieures et postérieures s'entrecroisent, s'entremêlent et parfois une région antérieure devient postérieure au simple changement d'activité qui s'y déroule. Par exemple, une part de la préparation de l'initiation se déroule sur le campus. Dès lors, un amphithéâtre peut servir à une réunion des différents comités organisateurs, et donc faire partie de la région postérieure ${ }^{17}$. Ensuite, ce même amphithéâtre peut servir à une activité durant l'initiation et cette fois appartenir à la région antérieure. Une fois encore, le contexte festif brouille la relation entre scène et coulisses, à tel point qu'il nous semble opportun de distinguer deux types de régions postérieures : celle où se prépare et s'organise l'initiation et celle qui est interdite, soit réellement, soit par consensus, par l'administration universitaire $^{18}$. De même, il faut spécifier trois régions antérieures. La première est publique ; les activités se déroulent à la vue de tous (espace public du campus, bar, etc.). La seconde est limitée ; il y a des observateurs extérieurs, mais ils sont en nombre restreint, dans le cas par exemple des activités se déroulant dans des pavillons réservés aux étudiants des programmes impliqués dans ces pavillons. Enfin, il y a la région antérieure limitée aux seuls participants : le site où se déroule la

17. En prenant pour acquis, en suivant Goffman, que la préparation se déroule en région postérieure.

18. Les bureaux administratifs ou de professeurs, par exemple. 
journée du hors-campus ou ceux des soirées privées en sont des exemples. En fait, les rôles vont, en partie, se définir selon l'accès à ces diverses régions. Les nouveaux évoluent dans l'ensemble des régions antérieures puisqu'ils sont partie prenante des activités qui s'y déroulent, mais n'ont pas accès aux régions postérieures dans le cadre de l'initiation. Les initiateurs ont accès à l'ensemble des régions, sauf la deuxième région postérieure. Les spectateurs n'ont, quant à eux, accès qu'à la première région antérieure et éventuellement à la seconde, selon leur statut. Enfin, les formes limitées et restreintes des régions antérieures sont différentes selon les programmes. En effet, une région antérieure limitée et restreinte sera accessible aux acteurs de l'initiation qui s'y déroule, mais elle ne le sera pas pour d'autres groupes d'initiés ou d'initiateurs d'autres programmes. Par contre, la région antérieure publique est aussi celle que se partage l'ensemble des programmes universitaires qui organisent une initiation ${ }^{19}$. Si l'accessibilité des régions définit en partie les rôles, le comportement de chacun est prépondérant. La conduite des initiés et des initiateurs dans les régions antérieures ressemble beaucoup aux attitudes de coulisses présentées par Goffman. Tout comme dans les bals, le contexte festif peut expliquer en partie ce débordement de comportement en dehors des régions postérieures. D'ailleurs, la région restreinte se rapproche fortement d'une forme de coulisses, à l'instar de l'après-bal, à ceci près qu'une représentation qui met en jeu deux équipes s'y déroule, contrairement à l'après-bal. Cependant, cette confusion entre coulisses et scène participe elle-même à la définition de la situation.

\section{La rupture de la représentation}

Les interactions sont donc reliées à la représentation des acteurs ou groupes d'acteurs selon les régions où ils évoluent. En fait, ces interactions visent à maintenir une représentation adéquate et sa définition. Toutefois, une représentation peut se détériorer rapidement, principalement de deux façons : par la révélation d'un secret ou par une conduite inappropriée d'un membre de l'équipe. Lorsqu'un membre rompt la représentation, ses coéquipiers tentent de la maintenir au mieux. Aussi ne réprimandent-ils pas le fautif immédiatement, mais ils attendent plutôt le retour en coulisses. "Des sanctions punitives immédiates auraient, bien au contraire, pour seul effet de troubler davantage encore

19. Ce qui ne veut pas dire qu'ils se la partageront en même temps. 
l'interaction et [...] de révéler au public un point de vue qui devrait être réservé aux seuls équipiers " (Goffman 1973 : 90). Dans une situation festive, autant les acteurs que le public comprennent que la représentation peut prendre des allures inattendues, puisque les limites permises y sont plus malléables : ce qui ne veut pas dire pour autant qu'il n'en existe pas. Prenons un exemple, le rapport à la personne ivre. Dans un contexte ordinaire, une personne ivre sera mal perçue par ses coéquipiers comme par le public. Mais lors des bals et des initiations, l'ivresse est permise, attendue, voire sollicitée (dans certaines épreuves). Le rapport à la personne ivre est donc différent, autorisé au sein du groupe mais selon certaines limites. Certaines personnes peuvent néanmoins détériorer la fête, par exemple ceux qui ne joueraient pas le jeu. Dans les bals et dans les initiations, le volontariat permet de contrer ce problème. Personne n'est obligé de participer. Cela est marquant dans les initiations, où le désistement des nouveaux au cours des premiers jours de l'initiation est relativement important. Dans les bals, le phénomène est moins courant mais il existe aussi, lorsque certains finissants quittent le bal plus tôt pour aller fêter en petits groupes en privé.

La notion de secret est également importante dans la théorie d'Erving Goffman. Il la présente comme ceci :

[1]es sujets que le public évite par respect pour l'acteur sont ceux-là mêmes qui rendraient l'acteur honteux s'ils venaient à être dévoilés. [...] Le public pressent des mystères et des pouvoirs secrets derrière la représentation, et l'acteur a l'intuition que ses secrets les plus importants sont dérisoires. Comme le montrent d'innombrables contes populaires et d'innombrables rites d'initiation, le véritable secret caché derrière le mystère, c'est souvent qu'en réalité il n'y a pas de mystère; le vrai problème, c'est d'empêcher le public de le savoir aussi (1973a: 71).

Il distingue plusieurs formes de secret, dont nous retiendrons deux, plus pertinentes à notre propos: les secrets inavouables d'une part « ils consistent en des faits concernant une équipe, des faits qu'elle connaît, qu'elle cache et qui sont incompatibles avec l'image d'ellemême et qu'elle s'efforce de maintenir devant son public » (1973a : 137) — et les secrets d'initiés d'autre part — « [c]e sont ceux dont la possession marque l'appartenance d'un individu à un groupe et contribue à ce que le groupe se sente distinct et différent de ceux qui ne sont pas dans le secret»(1973a: 138). Dans les bals de finissants, tout au long 
de la soirée, plusieurs mystères sont élucidés : les vêtements, la décoration de la salle, l'animation, etc., sont autant d'éléments qui demeurent cachés jusqu'à la dernière minute. Des mystères qui font partie de la représentation elle-même : ils n'entachent pas, au contraire, l'image de l'équipe «finissants ». Par contre, il y a autour de l'après-bal une aura de mystère d'autant plus intéressante que les médias dissertent souvent sur ce qui s'y déroulerait. L'idée de secret entourant l'après-bal est renforcée, d'autant plus que, depuis plusieurs années maintenant, les organisateurs ne dévoilent le lieu de l'événement qu'au tout dernier instant, voire même pas du tout. Mais ne peut-on y voir une forme de secret d'initiés, au moins temporaire ? En effet, durant toute la durée de l'après-bal, les finissants sont les seuls ${ }^{20}$ à savoir où ils sont et à partager ce secret. De plus, ils vont souvent rester évasifs sur ce qui s'y est réellement déroulé. Ce n'est certes pas un grand mystère, mais en conservant certains secrets, même relatifs, les finissants confirment que cet instant est à eux et à eux seuls.

Dans les initiations universitaires, il n'y a pas vraiment de secret, au plus une aura de mystère. Dans le cahier d'initiation « médecine 2002 », par exemple, un doute plane toujours autour des activités, particulièrement celle du hors-campus (dont le lieu, il est vrai, est tenu secret pour les nouveaux). Rien n'empêche cependant un ancien de raconter son initiation à un nouveau qu'il connaît, même si certains jouent le jeu et ne dévoilent rien ou presque, comme nous l'explique une initiée qui connaissait une ancienne.

Elle m'a pas dit grand chose, elle voulait pas me le dire. Mais ce qu'elle m'a dit, c'est que ce qui est vraiment chien, c'est qu'ils vont te faire faire un travail, ça fais-le pas (A.M.D.).

\section{Conclusion}

Cette lecture goffmanienne des bals de finissants et des initiations universitaires témoigne du dynamisme de ces rites par le regard posé sur les interactions. Le rituel se définit et se comprend à travers les relations qui s'établissent entre les différents acteurs. En ce sens, cette brève analyse conduit à une meilleure compréhension des rôles (donc des équipes) à travers leurs interactions (rites de ratification, par exemple), leur situation dans le rite, leur situation spatiale (les régions)

20. Il est en fait probable que quelques responsables scolaires soient dans le secret, mais pas toujours. 
ou non et par le contexte spécifique du rituel (festif, ludique). Ces différents éléments permettent, en définitive, de mieux saisir la distinction entre les bals de finissants et les initiations universitaires; les premiers impliquant davantage d'équipes (parents, administration scolaire, etc.) alors que les secondes sont beaucoup plus un rite à deux équipes : les initiés et les initiateurs. En somme, s'ils se rejoignent dans les grands traits de la ritualité, les bals de finissants et les initiations universitaires se distinguent plus l'un de l'autre qu'ils ne se ressemblent. Même leurs points communs, comme le port d'un costume, divergent (beaux habits d'un côté, dérision de l'autre). Les écarts sont profonds et s'inscrivent même dans les interactions qui ont cours entre les participants à l'intérieur d'un groupe et entre les différents groupes. Le rapport à l'espace et aux régions, selon les concepts goffmaniens, varie lui aussi, jouant sur une liaison confuse entre régions antérieures et postérieures. Si c'est le cas dans les bals comme dans les initiations, les premiers s'articulent davantage autour de la région antérieure, alors que les secondes s'expriment plutôt dans l'imprécision des frontières des différentes régions.

Une fois encore, le présent article n'est qu'un aperçu de ce que pourrait être une analyse approfondie des bals de finissants et des initiations universitaires sous la loupe goffmanienne. L'analyse des interactions individuelles, qui demanderait un travail considérable d'enquêtes et d'observations de la part du chercheur, serait certainement une voie très enrichissante à suivre. 


\section{Références}

Collection Martine Roberge (2000), ms 1. Archives de folklore et d'ethnologie de l'Université Laval.

Collection Martine Roberge et Karine Laviolette (2000), ms 1. Archives de folklore et d'ethnologie de l'Université Laval.

Harvengt, David, 2000-2004, enregistrements sur support mini-disque, entrevues auprès de participants aux initiations à l'Université Laval, collection privée.

Goffman, Erving, 1973a, La mise en scène de la vie quotidienne. 1. La présentation de soi. Paris, Éditions de Minuit.

$\ldots$ - 1973b, La mise en scène de la vie quotidienne. 2. Les relations en public. Paris, Éditions de Minuit.

Piette, Albert, 1988, Les jeux de la fête. Paris, Publications de la Sorbonne. 